\title{
Buchbesprechungen
}

1. R. S. Westfall, Force in Newton's Physics. 579 Seiten. Macdonald, London; American Elsevier, New York. £ 10.-.

Newton hat seiner Entwicklung der Mechanik drei Bewegungsgesetze zugrunde gelegt und dazu bemerkt, daß die beiden ersten das Ergebnis der Forschungen seiner Vorgänger seien. Insbesondere habe Galilei mit ihrer Hilfe die Wurfparabel hergeleitet. Wenn nun das erste Gesetz, das Trägheitsgesetz, mit Recht auf Galilei zurückgeführt werden kann, so trifft dies beim zweiten Gesetz nicht zu. Dieses lautet: «Die Änderung der Bewegung ist zur bewegenden Kraft proportional; sie erfolgt in derjenigen Richtung, in der die Kraft wirkt. »Vor Newton hat niemand ein solches Gesetz formuliert. Da nun zwischen dem Erscheinen der Discorsi des Galilei und Newtons Principia ein halbes Jahrhundert liegt, in dem sich so bedeutende Mathematiker wie Descartes und Huygens um die Gesetze der Mechanik bemühten, muß man schließen, daß es sehr schwierig war, dieses Grundgesetz der Mechanik aufzufinden.

Im vorliegenden Buch werden diese Schwierigkeiten untersucht. Der Verfasser schildert die wissenschaftliche Entwicklung, die zum Newtonschen Kraftbegriff geführt hat, ausführlich und verständnisvoll. Dabei wird deutlich, wie kompliziert die Fragestellung für die Gelehrten des 17. Jahrhunderts war. Das Ziel war es, ein mechanisches Weltbild zu entwerfen. Dabei schwebte den Gelehrten ein Begriff der Mechanik vor, der stark durch die Statik, durch «Hebel und Schrauben » geprägt war. Seit Descartes, für den die Materie mit dem Raum identisch war, sollten die Grundbegriffe zudem geometrische Begriffe sein. Denn diese allein kann man sich «klar und deutlich » vorstellen und damit auch mathematisch fassen. Der Newtonsche Kraftbegriff ist nun zwar mathematisch faßbar. Er ist aber weder geometrisch «klar und deutlich » vorstellbar, noch kann er auf einen «Mechanismus » zurückgeführt werden. Er erklärt also gerade das nicht, was die «mechanische Philosophie » erklären sollte, und er wurde darum auch von den Cartesianern - selbst von Huygens - abgelehnt, denen er als eine neue qualitas occulta erschien. Wenn es uns zurückblickend so scheint, als ob schon die Vorgänger Newtons nicht weit von seiner Einsicht entfernt gewesen wären, so entspricht dies der historischen Situation keineswegs. Die einen hatten zwar Vorstellungen, die denen Newtons ähnlich sind, die sie aber mathematisch nicht fassen konnten. Die anderen fanden mathematische Formulierungen, die denen Newtons gleichen, die sie aber in einer ganz anderen, "mechanischen» Weise, zu deuten strebten. Daß das Newtonsche Grundgesetz ganz allgemein jeder mechanischen Untersuchung zugrunde gelegt werden kann, ist ja auch erst durch die Bemühungen Eulers deutlich geworden. 
Der Verfasser stellt diese Entwicklungen und Verwicklungen in allen Einzelheiten dar, denn er verfügt über eine ausgebreitete und tiefe Kenntnis der Quellen. Die begriffliche Problematik steht dabei im Zentrum der Betrachtung. Daß die Principia nicht zuletzt epochemachend waren, wegen Newtons mathematischem Genie, ist dem Verfasser gewiß bewußt, kommt aber, seiner Zielsetzung wegen, nicht hinreichend zur Geltung. Das soll nicht als Kritik gelten, denn das vorliegende Buch ist eine bedeutende und originelle Leistung, die für fernere Studien grundlegend sein wird. Dazu helfen auch reiche, gelehrte Anmerkungen und eine sehr vollständige Bibliographie, die über 14 Seiten umfaßt.

Markus Fierz

\section{Robert Fox, "The Caloric Theory of Gases" from Lavoisier to Regnault.} Oxford Clarendon Press 1971.

Schon während der alchemistischen Zeit war es üblich, gemeinsame Eigenschaften stofflich zu erklären, indem man annahm, es würde ein gemeinsames «Prinzip » vorliegen. Alle sauren oder basischen Stoffe sollten z. B. ein und dieselbe Ursäure bzw. Urbase enthalten. Auch während der Phlogistonepoche (1680-1790) argumentierte man so, denn das Phlogiston ist das metallische Prinzip. Die Idee, die Wärme würde durch eine Feuersubstanz (matière du feu) hervorgerufen, lag deshalb nahe. Lavoisier nannte sie «Caloric» und glaubte, daß es sich um eine gewichtslose hochelastische Substanz, wahrscheinlich ein Element handle, das befähigt sei, sich mit andern Elementen zu verbinden. Man stellte sich vor, daß beim Erwärmen einer Substanz deren kleinste Teilchen mehr und mehr Caloric aufnehmen würden, was schließlich zu den Erscheinungen des Schmelzens, Verdampfens und der Gasausdehnung führen würde. Bei exothermen chemischen Reaktionen, z.B. den Verbrennungsprozessen, sollte Caloric abgegeben werden und die Temperaturerhöhung und sogar Feuer auslösen.

Gegen Ende des 18. Jahrhunderts begann man mit Hilfe der calorimetrischen Methoden die Mengen Caloric, die beim Erwärmen oder Abkühlen übertragen werden, quantitativ zu messen. Es entstand eine hochentwickelte Meßtechnik, mit der die spezifischen Wärmen zahlreicher Elemente und Verbindungen bestimmt wurden, und eine ausgeklügelte Theorie, welche es erlaubte, die Resultate quantitativ zu deuten. Die wichtigsten Träger dieser Entwicklung waren die französischen Gelehrten während der Revolution, des Kaiserreiches und der nachfolgenden zwei Jahrzehnte: Laplace, Carnot, Berthollet, Gay-Lussac, Dulong, Petit und andere. In England waren Dalton, der Schöpfer der Atomtheorie, ein überzeugter Caloriker, in dessen Abhandlung: A new system of chemical philosophy (1810) die Gasteilchen abgebildet werden mit einem Strahlenkranz von Caloric. Wegen der einfachen Gesetze, die bei Gasen beobachtet wurden, standen diese sogenannten elastischen Flüssigkeiten im Vordergrund des Interesses. Man lernte 